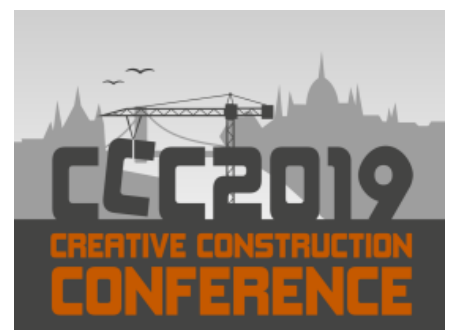

Available online at 2019.creative-construction-conference.com/proceedings/

CCC 2019

Proceedings of the Creative Construction Conference (2019) 116

Edited by: Miroslaw J. Skibniewski \& Miklos Hajdu

https://doi.org/10.3311/CCC2019-116

Creative Construction Conference 2019, CCC 2019, 29 June - 2 July 2019, Budapest, Hungary

\title{
Feasibility Study of Integrating BIM and 3D Printing to Support Building Construction
}

\author{
Kun-Chi Wang ${ }^{\mathrm{a}, *}$ and Mirosław Jan Skibniewski ${ }^{\mathrm{b}}$ \\ ${ }^{a}$ Chaoyang University of Technology, Taichung, Taiwan,wkc@cyut.edu.tw \\ ${ }^{b}$ University of Maryland, College Park,USA, mirek@umd.edu
}

\begin{abstract}
Although BIM has already been used to detect, simulate, and display construction details in many construction projects, these operations are performed and viewed exclusively on computers rather than on actual construction sites. In other words, when engineering staff holds meetings to discuss construction details, they are still required to use their imagination because they have no actual objects to refer to. Accordingly, they may be unable to correctly implement the construction details on actual sites, diminishing the benefits of BIM.

$3 \mathrm{D}$ printing technology is becoming increasingly mature. In recent years, the construction industry has also frequently discussed the use of 3D printing technology. Scholars have indicated that combining BIM and 3D printing technology will be one of the future directions for expanding the use of BIM. Nevertheless, when examining the application of 3D printing, the construction industry has often focused on large-scale printing (e.g., printing the entire house). By contrast, this study, which integrated BIM and 3D printing technology, used a small version of the 3D printing model to review operations that were difficult to discuss through imagination (e.g., how to solve interface conflicts and assemble precast components). By using this 3D printing model, engineering staff could examine and assemble actual components at the construction sites, thereby facilitating the construction work. In short, by utilizing the BIM to produce 3D printing models and components, and using said models and components as the basis for construction site-related discussions, engineering staff may be able to elevate the overall construction results.

This study examined whether integrating BIM and 3D printing technology was a feasible option for helping building design and construction. Furthermore, this study investigated how BIM could be used to quickly generate 3D printing models and the effectiveness of using 3D printing technology to print actual components during the construction process.
\end{abstract}

(C) 2019 The Authors. Published by Budapest University of Technology and Economics \& Diamond Congress Ltd.

Peer-review under responsibility of the scientific committee of the Creative Construction Conference 2019.

Keywords: 3D printing, building information modeling, construction details.

\section{Introduction}

On-site construction projects require the engineering staff to discuss numerous details of construction implementation methods. Such negotiation involves topics such as interface conflict solutions, steel bar binding methods, precast component assembly, and beam casing methods. Failure to address in advance the construction details for on-site implementation may result in progress delay and cost increase.

*Corresponding author: Kun-Chi Wang, email: wkc@cyut.edu.tw 
Kun-Chi Wang and Mirosław Jan Skibniewski/ Proceedings of the Creative Construction Conference (2019) 116 https://doi.org/10.3311/CCC2019-116

The rapid advancement of computer information and communication technology in recent years enables building data to be digitalized through building information modeling (BIM) based on a basic three-dimensional (3D) structure. Such modeling can automatically generate geometric and nongeometric parameter characteristics of building components, thereby gradually drawing attention from the engineering industry and academia (Chen, 2009; Guo and Hsieh, 2010; Zhang and Hu, 2011). Numerous construction projects have benefited from the assistance of BIM by conducting assessments, simulations, and demonstrations of construction details on computers. However, the model has yet to be physically implemented. That is, engineering crews still must rely on partial guesswork when attempting to decide the implementation details during meetings. Lacking a sense of concreteness, engineering crews may not be able to follow precise procedures on-site, thereby limiting the benefits of BIM.

The technology of 3D printing has become increasingly mature. Because of its high degree of customization, fixed cost, and short completion times, 3D printing technology has been widely used in the construction industry in addition to the medical and manufacturing industries. However, past discussions of the aforementioned combination have usually focused on large-scale printing (e.g., printing an entire house) as the research direction, whereas this study used miniature 3D models to examine the actual implementation steps of construction projects that are difficult to visualize during discussions, such as addressing interface conflicts and precast component assembly methods. Miniature 3D printing models enable the actual assembly or examination of components on a construction site, effectively assisting in the process of construction. That is, if the BIM models and components can be output as 3D-printed models as the basis for discussion on the construction site, the overall construction project performance should be improved.

Therefore, this study explored the feasibility of integrating BIM with 3D printing technology to assist in the design and construction of buildings. In addition to exploring how to 3D print models rapidly from BIM models, the present study also investigated the effectiveness of 3D-printed solid components on improving the convenience of project implementation.

\section{Literature Review}

\subsection{State of $3 D$ printing}

The technology of 3D printing originated from rapid prototyping technology. In 1981, two 3D-printing methods were first proposed. In 1984, US 3D Systems founder, Chuck Hull, developed 3D rapid prototyping technology. The first patented 3D printing technology was applied by the Massachusetts Institute of Technology in 1989. It was not until 2010 that related applications of 3D printing began to develop.

The present applications of 3D printing are only mildly customizable and focus particularly on unique shapes and products that are difficult to process through conventional techniques. Future businesses may adopt high-value but a low quantity of tech products. In terms of applications of 3D printing in engineering projects, numerous laboratories have begun to adopt the technique for small objects (Misao, 2014). Apis Cor, a San Francisco-based startup company, printed a complete circular building including a living room, a bathroom, and a bedroom in less than 24 hours using concrete; the building cost approximately US\$10,000 (Tu, 2017). Behrokh (2012) also revealed that 3D printing will eventually be able to complete building construction in less than 20 hours. The incorporation of 3D printing techniques can substantially reduce the production time of building components. Using printed components as references, engineering crews may discuss precast component assembly methods, steel bar binding methods, and interface conflict solutions during their meetings.

\subsection{Applications of BIM and $3 D$ models in construction projects}

Chen and Tsai (2015) assisted in the maintenance and management of construction projects using 3D printing. They developed a 3D printing-based building maintenance system, in which building information is stored in advance in a database. Subsequently, a platform they developed was used to extract parameter information to establish a 3D printing model of a building, which was then printed for assembly. 
Kun-Chi Wang and Mirosław Jan Skibniewski/ Proceedings of the Creative Construction Conference (2019) 116 https://doi.org/10.3311/CCC2019-116

You (2017) combined virtual BIM and a 3D solid model for virtual and physical integration. The 3D model of that study project was obtained through fused deposition modeling with polylactic acid as the material. However, some components in the study project still had loose joints. Some components still could not be 3D printed and thus were replaced with other materials such as fishing lines.

\section{Method of integrating BIM model and 3D Printing}

Although BIM has already been used to detect, simulate, and display construction details in many construction projects, these operations are performed and viewed exclusively on computers rather than on actual construction sites. In other words, when engineering staff holds meetings to discuss construction details, they are still required to use their imagination because they have no actual objects to refer to. Accordingly, they may be unable to correctly implement the construction details on actual sites, diminishing the benefits of BIM.

3D printing technology is becoming increasingly mature. In recent years, the construction industry has also frequently discussed the use of 3D printing technology. Scholars have indicated that combining BIM and 3D printing technology will be one of the future directions for expanding the use of BIM. Nevertheless, when examining the application of 3D printing, the construction industry has often focused on large-scale printing (e.g., printing the entire house). By contrast, this study, which integrated BIM and 3D printing technology, used a small version of the 3D printing model to review operations that were difficult to discuss through imagination (e.g., how to solve interface conflicts and assemble precast components). By using this $3 \mathrm{D}$ printing model, engineering staff could examine and assemble actual components at the construction sites, thereby facilitating the construction work. In short, by utilizing the BIM to produce 3D printing models and components, and using said models and components as the basis for construction siterelated discussions, engineering staff may be able to elevate the overall construction results.

\section{Case study}

This study took a steel-pipe dam as an example and output a 3D-printed model from BIM to evaluate the feasibility of combining BIM with 3D printing, thereby investigating the benefits of 3D printing for construction projects. The steelpipe dam utilized a new construction method. Precast steel pipes were assembled on the river during construction to maximize the convenience of installation. This study 3D printed a model of the pipes of the dam and discussed the assembly method in advance to ensure the efficiency of on-site assembly.

Testing revealed that the 3D solid model might not be able to present the details of the engineering facilities if the model scale was reduced. An excessively large output scale (e.g., 1:50) would require the printing process to be split into multiple parts to display facility details. Moreover, storing large 3D models may be a problem. Therefore, the appropriate printing scale for 3D models is a topic that requires further discussion. The printing procedure of converting BIM components into a solid 3D model involves the following nine steps. The BIM model of the steel dam is shown in Figs 1 and the printed steel pipe in this study is presented in Figs. 2-3.

(1) Establish a highly precise BIM model for soil and water conservation projects (including engineering facilities and topography).

(2) Launch Revit and export the Revit file.

(3) Import the Revit file into 3ds Max, from which the stereolithography (STL) print file is exported.

(4) Import the STL file into Autodesk 123D:

a、 Perform model patching: Fix, check, and repair the loose joints and errors in the model.

b、 Set the model output scale.

c、 Cut the model according to the 3D printer's output scale; each model after cutting is output as an individual STL file.

d、 Examine each STL file and select the optimal model plane as the bottom for printing. All files are saved afterward.

(5) Calibrate and warm up the 3D printer, and then set the print quality. 
Kun-Chi Wang and Mirosław Jan Skibniewski/ Proceedings of the Creative Construction Conference (2019) 116 https://doi.org/10.3311/CCC2019-116

(6) To avoid the wires prepared by hot melt extrusion failing to stick firmly to the platform of the 3D printer during the printing process, this study placed temperature-resistant masking tape on the platform and applied glue from a glue stick to increase the adhesion of the wire.

(7) Select an STL file to conduct a printing test.

(8) Examine the quality of the solid 3D model after printing. If the crucial details cannot be observed, the printing scale must be adjusted (i.e., the fourth step must be repeated). The printing test is performed repeatedly until the model meets the requirements.

(9) Print the BIM components according to the appropriate scale determined by the printing test.

However, the proposed method of converting BIM models into 3D-printed models still requires modifications because it could not rapidly generate the required models through $3 \mathrm{D}$ printing.

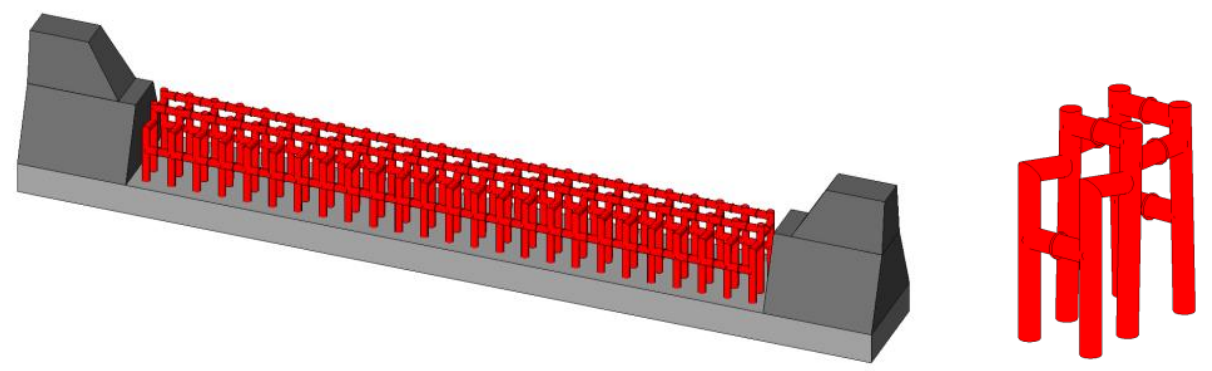

Fig. 1. (a) BIM model of steel-pipe dam $\quad$ (b) BIM model of steel pipe.

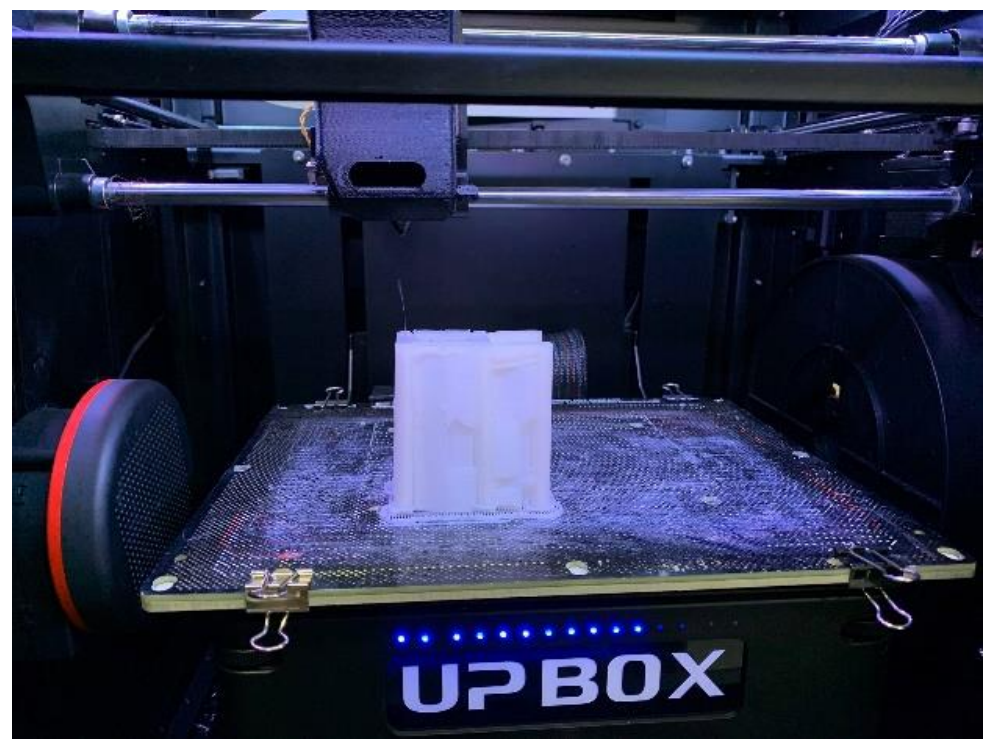

Fig. 2. printing the 3D model of the steel-pipe dam. 
Kun-Chi Wang and Mirosław Jan Skibniewski/ Proceedings of the Creative Construction Conference (2019) 116 https://doi.org/10.3311/CCC2019-116
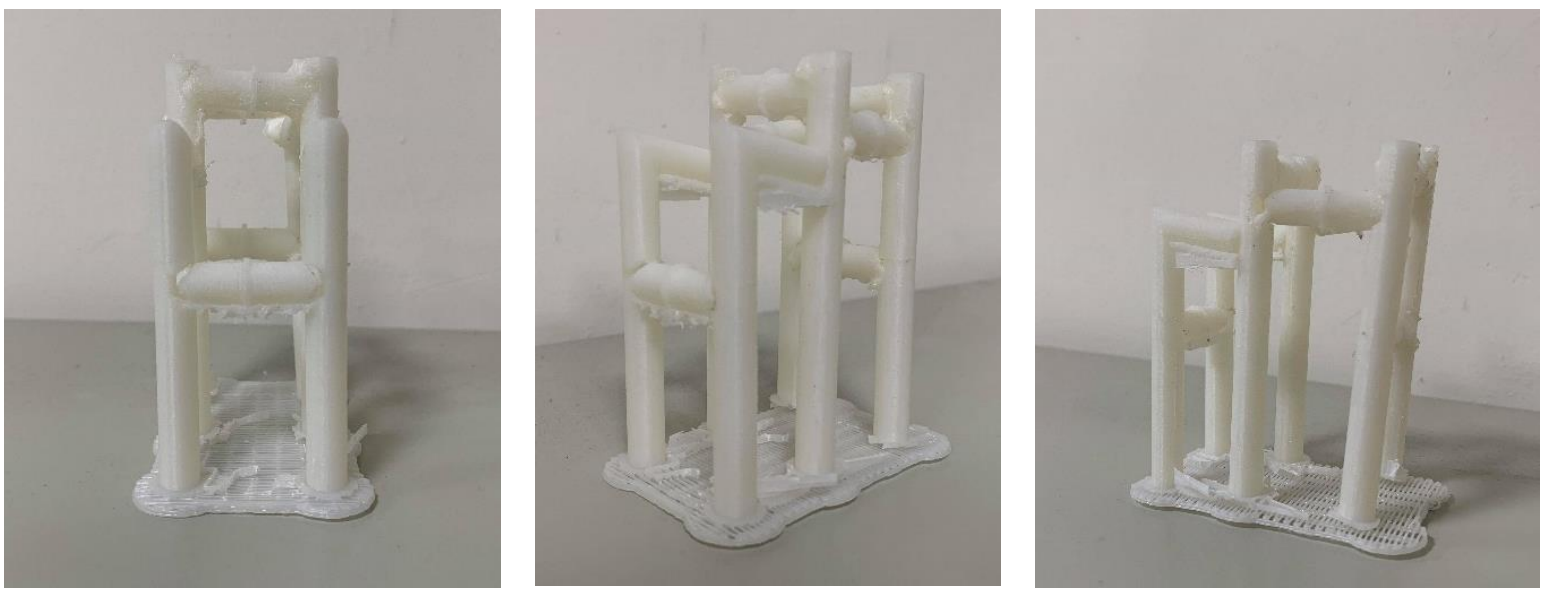

Fig. 3. part of 3D printed steel pipe.

\section{Conclusion and Suggestion}

This study attempted to integrate BIM with 3D printing to explore a method for converting building data into 3D models using BIM software, Revit. The obtained model was conducive to evaluating the feasibility of construction project implementation. The experiment in this study revealed that direct conversion of BIM ideas into a 3D model may reduce the time required for 3D model construction and may allow engineers to perform model modifications and printing directly.

\section{Acknowledgements}

This research is financially supported by the Ministry of Science and Technology of Taiwan (Contract No. MOST 107-2218-E-324-004-). The authors would like to sincerely express our appreciation to professor Wei-Chih Wang in National Chiao Tung University for the recommendations in this research.

\section{References}

[1] CIC, (2011). BIM Project Execution Planning Guide-Version 2.0, Computer Integrated Construction (CIC) Research Program, The Pennsylvania State University, University Park, PA, USA. Available at: https://vdcscorecard.stanford.edu/sites/g/files/sbiybj8856/f/ bim_project_execution_planning_guide-v2.0.pdf. [Accessed 07 Mar. 2019]

[2] J. P. Zhang, and Z. Z. Hu, "BIM- and 4D-based integrated solution of analysis and management for conflicts and structural safety problems during construction: 1. principles and methodologies," Automation in Construction, 20, 2011, pp. 155-166. doi: 10.1016/j.autcon. 2012.05.008.

[3] K. C. Wang, M. C. Ho, W. C. Wang, "Applying BIM Quantity Takeoffs to Support Conceptual Cost Estimation for Public Construction Projects," Construction Management Journal, 104, (2016) pp. 1-13. doi: 10.22260/ISARC2018/0040

[4] C. S. Tu, Apis Cor creates the first 3D printed home in 24 hours, 2017. Access on: https://www.digitimes.com.tw/iot/article.asp?cat=158\&cat1 $=20 \&$ cat $2=10 \& i d=0000497754 \_m d j 67 c h h 6 p 3 t 2 m 306 s 621$. [Accessed 15 Mar. 2019]

[5] Misao, The concept, principle and application of 3D printing: a complete understanding of the new manufacturing technology that is about to change the world, 2014, Muma Culture. ISBN : 9789863590521.

[6] R. C. Guo and S. S. Hsieh, BIM Overview and Domestic Implementation Strategy, The Magazine of The Chinese Institute of Civil and Hydraulic Engineering, 37(5) 2010 pp. 8-20. Available at: https://www.academia.edu/4739780/\%E5\%9C\%9F\%E6\%9C\%A8\%E6\%B0\%B4\%E5\% 88\%A9_BIM\%E6\%A6\%82\%E8\%AA\%AA\%E8\%88\%87\%E5\%9C\%8B\%E5\%85\%A7\%E6\%8E\%A8\%E8\%A1\%8C\%E7\%AD\%96\%E7\%95 \%A5_\%E9\%83\%AD\%E6\%A6\%AE\%E6\%AC\%BD_\%E8\%AC\%9D\%E5\%B0\%9A\%E8\%B3\%A2. [Accessed 15 Mar. 2019]

[7] Y. J. Chen, The Development of Project Information Model and the Applications of PIM Model-based System, Master's Thesis, Department of Civil Engineering, National Cheng Kung University, Tainan, Taiwan, 2009 (in Chinese). Available at: https://ndltd.ncl.edu.tw/cgibin/gs32/gsweb.cgi/login?o=dnclcdr\&s=id=\%22097NCKU5015089\%22.\&searchmode=basic. [Accessed 05 Dec. 2018]

[8] M. H. Chen and J. T. Tsai, Using 3D Printing to Assist Building Maintenance and Preservation, FCU ePaper, Department of Information Engineering and Computer Science, Feng Chia University, Taichung, Taiwan, 2015 (in Chinese). Available at: http://dspace.lib.fcu.edu.tw/ bitstream/2377/31545/1/D0224718104101.pdf [Accessed 01 Mar. 2019]

[9] Y. S. You, Research on the Application of BIM and 3D Printing Technology in the Construction of Engineering Model, Master's Thesis, Graduate Institute of Civil Engineeting and Hazard Mitigation Design, China University of Technology, Taipei, Taiwan, 2017 (in Chinese). 
Kun-Chi Wang and Mirosław Jan Skibniewski/ Proceedings of the Creative Construction Conference (2019) 116 https://doi.org/10.3311/CCC2019-116

Available at: https://ndltd.ncl.edu.tw/cgi-bin/gs32/gsweb.cgi/login?o=dnclcdr\&s=id=\%22105CKIT0653005\%22.\&searchmode=basic. [Accessed 01 Mar. 2019] 